\title{
Education and Research in Public Policy
}

\author{
Keiko Nakayama, Masatoshi Shirai \\ Faculty of Economics, Chukyo University, 101-2, Yagotohonmachi, Showa, Nagoya, 466-8666, Japan
}

Email address:

nakayama@mecl.chukyo-u.ac.jp (K. Nakayama)

\section{To cite this article:}

Keiko Nakayama, Masatoshi Shirai. Education and Research in Public Policy. International Journal of Economic Behavior and Organization. Special Issue: Recent Developments of Economic Theory and Its Applications. Vol. 3, No. 2-1, 2015, pp. 46-51.

doi: 10.11648/j.ijebo.s.2015030201.18

\begin{abstract}
This paper constructs an endogenous growth model where the educational sector (higher education) produces human capital and social knowledge by education and research activities. The steady state growth paths are studied for market economy where the educational sector is financed by the income tax imposed on household and the rent on use of social knowledge by firm. We show that the education-research allocation in the educational sector determines the income tax rate and also the growth rate for the market economy. We conclude that there exists a certain education-research allocation maximizing the steady state growth rate, and that the optimal income tax rate is not necessarily zero.
\end{abstract}

Keywords: Human Capital, Social Knowledge, Education

\section{Introduction}

Recently, the stress has been on the role of education in endogenous growth models. Eckstein and Zilcha (1994) and Zhang (1996) show that the public education system can increase the growth rate of the society when human capital has the nature of externalities. Lucas (1988) points to human capital accumulation as an important engine of growth. Furthermore, Romer (1990) analyzes that the endogenous technological change created by R\&D activity determines the growth rate in the economy.

While both education and research affect the growth rate through human capital and technological change, they are dealt with separately in the literature. However, in fact, they are produced simultaneously by the higher educational institution, for example, university. Furumatsu and Shirai(1998), Kitaura and Shirai(2012) analyze how the educational sector (university) contributes to a whole society through education (teaching) and research activities, pointing out that human capital and social knowledge are produced jointly. Since social knowledge produced by research activity in the educational sector has a public nature, the market cannot achieve the efficient resource allocation without government intervention. According to Ministry of Education, Culture, Sports, Science and Technology-JAPAN(2013), in many developed countries, a quarter of the public budget is devoted to education and research in the educational sector.

Stressing the importance of the stock of infrastructure (non-rival goods) in economic growth, Dasgupta $(1999,2001)$ analyzes the dynamic growth equilibrium of the market economy where the production of infrastructure is financed out of the sale of infrastructure services and the tax on profit income. He derives the result that the optimal income tax is zero and concludes that the market economy grows faster than the command economy even though the social welfare achieved is dominated by the latter.

Our purpose is to reconstruct an education-research model in a continuous growth setting like Dasgupta and analyze the optimal growth rate in the market economy where the educational sector is financed by the tax imposed on the household income and the rent on use of social knowledge. We assert that the education-research allocation in the education sector determines the tax rate and the growth rate in equilibrium, and conclude a certain education-research allocation will maximize the steady state growth rate, and that the optimal income tax rate is not necessarily zero.

\section{Model Preliminaries}

We consider a two-sector economy, which consists of a production sector and an educational sector.

The production sector manufactures one kind of a consumption goods using effective labor (measured by human capital) and knowledge. The production function of consumption good is defined as

$$
Y=A h_{Y}{ }^{\alpha} H^{1-\alpha}, 0<\propto<1,
$$


where $Y$ stands for the production quantity of consumption goods, $h_{Y}$ and $H$ are the stocks of human capital and social knowledge used to produce $Y$, respectively. 1 The constant term $A$ is parametrically specified and positive. Social knowledge is assumed to be a non-excludable and non-rival commodity.

In the educational sector, two types of educational outputs, the additional human capital and social knowledge, are produced by education and research activities. We assume that the portion of those educational outputs can be determined by the allocation of human capital between two activities. We define $\lambda$ as the proportion of human capital devoted to education activity, and then $1-\lambda$ is the proportion devoted to research activity. The production function in the educational sector is formulated as follows:

$$
\begin{gathered}
\dot{h}=B\left(\lambda h_{E}\right)^{\beta} H^{1-\beta}, \\
\dot{H}=B\left[(1-\lambda) h_{E}\right]^{\beta} H^{1-\beta}, \\
0<\beta<1, \\
0<\lambda<1,
\end{gathered}
$$

where $\dot{h}$ and $\dot{H}$ are respectively the change in the stock of human capital, $\dot{H}$ and the social knowledge, $h_{E}$ is the stock of human capital used in the educational sector, and the constant term $B$ is parametrically specialized and positive. 2 Human capital is accumulated in household through the educational process, while the social knowledge is accumulated as the intellectual common wealth in the society as a whole.

We consider a representative household, which lives infinitely and has the following lifetime CES utility function:

$$
U=\int_{0}^{\infty} \frac{c(\theta)^{1-\sigma}}{1-\sigma} e^{-\rho \theta} d \theta
$$

where $\mathrm{c}(\theta)$ is he consumption at time $\theta$, the constant $\rho>0$ is the discount parameter, and $\sigma>0$ is the elasticity of instantaneous marginal utility. We assume that the social welfare is identical with household utility.

\section{Static Equilibrium}

We examine the static equilibrium in the market economy, where total human capital stock and social knowledge stock are fully employed in the society. As noted in the previous section, the production sector can use, as public goods, social knowledge produced by the educational sector. We suppose that government can impose the rent $r$ on a unit use of social knowledge and spends the rent revenue on the educational sector.3 Education (human capital) is supposed to be publicly supplied free of charge, whose costs are financed through the rent revenue and a proportional tax on the wage income. 4

\footnotetext{
1 While Furumatsu and Shirai (1998) consider the externalities of human capital in the process of production, we disregard this aspect of human capital here.

2 Both parameters B and $\beta$ are assumed to be the same in the two activities for simplification.

3 We suppose that this rent corresponds to a payment such as patent fees or copyright.

4 Alternatively, we can consider the tax imposed on household income as tuition
}

We suppose that a representative firm in the production sector maximizes profit assuming all factor prices to be given. For the firm to behave competitively, the ratio of marginal products of the factors should be equal to their price ratio. This means

$$
h_{Y}=\frac{\alpha}{1-\alpha} \frac{r}{w} H
$$

where $w$ is the wage rate. Given the in elastically supplied value of $H$, (5) defines the firm's demand for human capital $h_{Y}$ as a function of $r / w$ when $H$ is fully used.

The educational sector produces human capital and social knowledge subjected to the balanced budget. The budget constraint is

$$
r H+\tau w h=w h_{E},
$$

where $\tau$ is the income tax rate and $h$ is total stock of human capital in the society. $5 h$ is assumed to be equal to the total supply of human capital. (6) can be rewritten as follows:

$$
h_{E}=\frac{r}{w} H+\tau h,
$$

which determines the demand for human capital by the educational sector $h_{E}$ as a function of $r / w$ for given $H, \tau$ and $h$.

Total demand for human capital is

$$
h=h_{Y}+h_{E} .
$$

This should be equal to total stock $h$ in (6). Substituting (5) and (7), total demand (8) is rewritten as follows:

$$
h=\frac{1}{1-\tau} \frac{1}{1-\alpha} \frac{r}{w} H .
$$

(9) determines the combinations of the relative price of social knowledge to human capital $(r / w)$ and the total demand for human capital $(h)$ consistent with a full utilization of the given stock of social knowledge. Then we have the following proposition.

Proposition 1. At any given point of time, each possible specification of the income tax rate and the stock of human capital and social knowledge gives rise to a unique positive ratio of prices such that the market for human capital is in equilibrium and the social knowledge is fully utilized.

Given the equilibrium prices ratio established by Proposition 1, the ratio $h_{Y} / H$ employed in the production sector is known from (5). Since the firm's technology displays constant returns to scale, the marginal productivity of each factor is known for the production sector. For competitive behavior, it is necessary that $w$ and $r$ are equal to the respective marginal productivities. Thus, the wage rate and the price of social knowledge are given by

$$
w=\alpha A\left(\frac{h_{Y}}{H}\right)^{\alpha-1}
$$

charged on human capital stock accumulated in the past.

5 The income tax is regarded as being imposed not only labor income but also on human capital. The wage rate is assumed to be equal in both sectors. 


$$
r=(1-\alpha) A\left(\frac{h_{Y}}{H}\right)^{\alpha}
$$

Moreover, we can derive monotonic relationships between the market clearing $w, r$ and $\frac{h}{H}$. From (5), (9), (10) and (11), we have

$$
\begin{gathered}
w=\alpha A\left((1-\tau) \alpha \frac{h}{H}\right)^{\alpha-1} \\
r=(1-\alpha) A\left((1-\tau) \alpha \frac{h}{H}\right)^{\alpha} .
\end{gathered}
$$

(12) and (13) give the wage rate and the price of social knowledge consistent with a full utilization of the stocks of human capital and social knowledge at any income tax rate, respectively.

Next, let us consider the allocation rule of human capital between two sectors. Since the production function of consumption goods is of a Cobb-Douglas type, we have the fact that the share of each factor in the production sector must be a constant under competitive conditions. Thus,

$$
\begin{gathered}
w h_{Y}=\alpha Y, \\
r H=(1-\alpha) Y .
\end{gathered}
$$

Furthermore, using these equations and the budget constraint of the educational sector we have

$$
\frac{h_{E}}{h_{\mathrm{Y}}}=\left(\frac{1}{1-\tau}\right)\left(\frac{1-\alpha}{\alpha}\right)+\frac{\tau}{1-\tau}
$$

(16) represents the allocation ratio of human capital between two sectors. However, this allocation rule is inefficient for the following two reasons. First, in the educational sector, the marginal product of human capital is not equal to the wage rate. Second, the price of social knowledge is not equal to social marginal products but its private one. The latter causes the under supply of social knowledge.

Differentiating (16) with respect to $\tau$, we obtain

$$
\frac{\partial\left(\frac{h_{E}}{h_{Y}}\right)}{\partial \tau}=\frac{\frac{1-\alpha}{\alpha}+1}{(1-\tau)^{2}}>0 .
$$

This means that a rise in the income tax rate expands the educational sector, and vice versa. This is because the rise in the tax rate reduces the rent-wage ratio to decrease employment of human capital in the production sector in equilibrium.

In this section, we considered the wage rate, the price of social knowledge, and the allocation rule of human capital between two sectors consistent with the stocks of human capital and social knowledge utilized fully for a given income tax rate at any point of time. Then, household's demand for consumption goods determines the static equilibrium. The problem remaining is how educationresearch allocation in the educational sector affects the work of economy. In the next section, we analyze the change in human capital stock consistent with one desired by the representative household in the dynamic steady state.

\section{Dynamic Steady State Equilibrium}

We define a state of steady growth as $h_{Y} / h$ and $h_{Y} / H$ to be constant, that is, $h / H$ is constant for all, and $\dot{H} / H=$ $\dot{h} / h=\dot{Y} / Y=\dot{c} / c$ to be satisfied and constant. Following Dasgupta (1999), we will call $\dot{H} / H$ the supply rate of growth of system $\left(g^{S}\right)$, since it imposes an upper bound on the growth rate achievable in the economy. Similarly, we will call $\dot{c} / c$ the demand rate of growth $\left(g^{d}\right)$. The dynamic steady state equilibrium occurs when $g^{s}=g^{d}$.

The supply rate of growth, $g^{s}$, is derived from (3) as follows:

$$
g^{S}=\frac{\dot{H}}{H}=(1-\lambda)^{\beta} B\left(\frac{h_{E}}{H}\right)^{\beta} .
$$

Using (5), (7), (8), (10) and (11) to rewrite (18), $g^{s}$ turns out to be

$$
g^{S}=(1-\lambda)^{\beta} B\left[\frac{1}{1-\tau}\left(\frac{1-\alpha}{\alpha}+\tau\right)\left(\frac{r}{(1-\alpha) A}\right)^{\frac{1}{\alpha}}\right]^{\beta} .
$$

The supply rate of growth is an increasing function of the price of social knowledge and the income tax rate, Intuitively, this result reflects the fact that a rise in the educational sector, thereby increases the growth rate of social knowledge.

Next, we consider the representative household's behavior. The household spends the after-tax income on consumption and accumulation of human capital. We assume that the household needs some given amounts of consumption goods to accumulate a unit of human capital, and, without the loss of generality, that this is unity 6 . The household chooses the amounts of consumption and the change of human capital so as to maximize the utility (4) subject to the instantaneous budget constraint:

$$
\mathrm{c}+\dot{h}=(1-\tau) w h
$$

This maximizing problem is expressed as

$$
\begin{aligned}
& \operatorname{Max} \int_{0}^{\infty} \frac{c(\theta)^{1-\sigma}}{1-\sigma} e^{-\rho \theta} d \theta \\
& \text { s.t. } c+\dot{h}=(1-\tau) w h
\end{aligned}
$$

We define the current value Hamiltonian $H_{h}$ as

$$
H_{h} \equiv \frac{c^{1-\sigma}}{1-\sigma}+\eta((1-\tau) w h-c),
$$

where $\eta$ is the costate variable. We obtain the first order conditions as follows:

$$
\begin{gathered}
c^{-\sigma}=\eta, \\
\frac{\dot{\eta}}{\eta}=\rho-(1-\tau) w .
\end{gathered}
$$

From (21) and (22), we obtain

6 This means that the household must purchase certain goods (textbook, references and so on) to accumulate a unit of human capital. We assume here that human capital is supplied freely. 


$$
\frac{\dot{c}}{c}=\frac{(1-\tau) w-\rho}{\sigma} .
$$

Also, we have from (10) and (11),

$$
w=\alpha A\left(\frac{r}{(1-\alpha) A}\right)^{\frac{\alpha-1}{\alpha}} .
$$

Substituting (24) to (23), $g^{d}$ turns out to be

$$
\begin{aligned}
g^{d}=\frac{\dot{c}}{c}=\frac{(1-\tau) \alpha A\left(\frac{r}{(1-\alpha) A}\right)^{\frac{\alpha-1}{\alpha}}-\rho}{\sigma} . \\
\quad(1-\lambda)^{\beta} B\left[\frac{1}{1-\tau}\left(\frac{1-\alpha}{\alpha}+\tau\right)\left(\frac{r}{(1-\alpha) A}\right)^{\frac{1}{\alpha}}\right]^{\beta}=\frac{(1-\tau) \alpha A\left(\frac{r}{(1-\alpha) A}\right)^{\frac{\alpha-1}{\alpha}}-\rho}{\sigma} .
\end{aligned}
$$

However, we should add another constraint to attain the steady state. It is caused by the fact that the ratio of product in the educational sector should be constant. From (2) and (3), we obtain

$$
h=\left(\frac{\lambda}{1-\lambda}\right)^{\beta} H .
$$

Since human capital h and social knowledge $H$ grow at the same rate on the steady state growth path, we have

$$
\frac{h}{H}=\left(\frac{\lambda}{1-\lambda}\right)^{\beta}
$$

Then, the ratio of human capital and social knowledge stocks in the steady state growth is determined by the technology in the educational sector. This is another constraint which should be imposed on the relation of the income tax rate and the price of social knowledge. (13) is rewritten as

$$
r=(1-\alpha) A\left((1-\tau) \alpha\left(\frac{\lambda}{1-\lambda}\right)^{\beta}\right)^{\alpha}
$$

In order for economy to be in true equilibrium, (29) is necessary to hold for the equilibrium price of social knowledge as well as (26). The steady state equilibrium is represented as a solution of a system of simultaneous (26) and (29). By substituting (29) to (26), we have

$$
(1-\lambda)^{\beta} B\left[\left(\frac{1-\alpha}{\alpha}+\tau\right) \alpha\left(\frac{\lambda}{1-\lambda}\right)^{\beta}\right]^{\beta}=\frac{[(1-\tau) \alpha]^{\alpha} \mathrm{A}\left(\frac{\lambda}{1-\lambda}\right)^{\beta(\alpha-1)}-\rho}{\sigma} .
$$

(30) gives the relation between $\lambda$ and $t$ that should hold in the steady state growth equilibrium. In order to investigate this relation, we rewrite (30) as follows:

$$
\begin{gathered}
g^{S}(\tau, \lambda)=(1-\lambda)^{\beta} B\left[\left(\frac{1-\alpha}{\alpha}+\tau\right) \alpha\left(\frac{\lambda}{1-\lambda}\right)^{\beta}\right]^{\beta} \\
g^{d}(\tau, \lambda)=\frac{[(1-\tau) \alpha]^{\alpha} A\left(\frac{\lambda}{1-\lambda}\right)^{\beta(\alpha-1)}-\rho}{\sigma}
\end{gathered}
$$

We illustrate the $g^{s}$ and $g^{d}$ curves in the $(\tau, g)$ plane in Figure 1. Obviously, $g^{s}$ is a monotone increasing function and $g^{d}$ is a monotone decreasing function of $\tau$ for any
(25) shows that the demand rate of growth is a decreasing function of the price of social knowledge and the income tax rate. The reason is that a rise in the income tax rate decreases household income, and a rise in the price of social knowledge decreases the wage rate from (24).

The equilibrium price of social knowledge is determined by equating (19) and (25):

$\lambda \in(0,1) . g^{s}$ curve approaches to the horizontal axis for any $\tau$ as $\lambda \rightarrow 0$ and $\lambda \rightarrow 1$, and shifts upward with $\lambda$ for $\lambda \in$ $(0, \beta)$ and downward for $\lambda \in(\beta, 1)$. Therefore, $g^{s}(\tau, \beta)$ is an upper bound of $g^{s}$. On the other hand, the $g^{d}$ curve approaches to the vertical line passing through $(1,-\rho / \sigma)$ as $\lambda \rightarrow 0$, and turns anticlockwise around $(1,-\rho / \sigma)$ with $\lambda$, and approaches to the horizontal line passing through $(1,-\rho / \sigma)$ as $\lambda \rightarrow 1$. We can obtain a point of intersection of the $g^{s}$ and $g^{d}$ curves for some $\lambda$ in nonnegative region on the $(\tau, g)$ plane. This point corresponds to the steady state growth equilibrium. However, we note that there is not always an equilibrium point for any $\lambda$. Since the income tax rate cannot be negative in our model, there is no equilibrium if the $g^{s}$ and $g^{\mathrm{d}}$ curves intersect on the left side of the vertical axis. For the equilibrium to exist that $g^{s}(0, \lambda)<g^{\mathrm{d}}(0, \lambda)$. $g^{s}(0, \lambda)>0$ for any $\lambda \geq 0$, but $g^{\mathrm{d}}(0, \lambda)<0$ for larger $\lambda$. Then we have the maximum value of $\lambda$ as $\bar{\lambda}$, which satisfies $g^{s}(0, \bar{\lambda})=g^{d}(0, \bar{\lambda})$. For any given $\lambda \leq \bar{\lambda}$, we can always find the steady state growth equilibrium. Then, we have the following proposition.

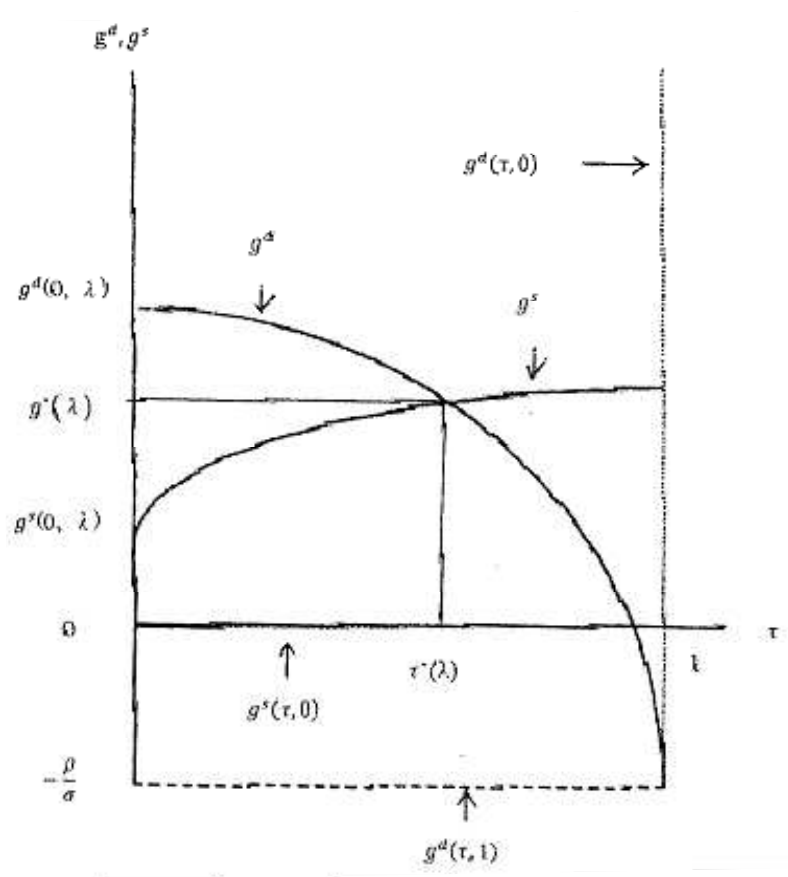

Figure 1. Equilibrium growth rate and tax rate 
Proposition 2. There exists a steady state growth equilibrium, which fully utilizes the stock of social knowledge for any education-research allocation less than or equal to $\bar{\lambda}$.

We examine how the equilibrium income tax rate and the growth rate change with $\lambda$. Starting from very small $\lambda, g^{s}$ curve shifts upward from horizontal axis with $\lambda$ until $\lambda$ reaches $\beta$, thereafter it shifts downward to the horizontal axis, on the other hand the $g^{d}$ curve always turns left with $\lambda$. The intersection of $g^{s}$ and $g^{d}$ curves moves to northwest with $\lambda$ when $\lambda$ is small, and begins to move southwest at a certain $\lambda$. We define the value of $\lambda$ at which the turning point occurs as $\lambda^{*}$. Therefore, the growth rate (the intersection of $g^{s}$ and $g^{d}$ curves) would continue to rise with $\lambda$ up to $\lambda^{*}$, which means that the growth rate reaches maximum value at $\lambda^{*}$. However, we cannot choose any $\lambda$ larger than $\bar{\lambda}$ for the equilibrium. If $\bar{\lambda} \leq \lambda^{*}$, then we can obtain the maximum equilibrium growth rate by choosing the education-research allocation $\bar{\lambda}$ and imposing zero income tax rate. On the contrary, if $\bar{\lambda}>\lambda^{*}$, then we can obtain the maximum growth rate by setting the education-research allocation to $\lambda^{*}$ and imposing a positive income tax rate corresponding to $\lambda^{*}$. Further, we can show that $\lambda^{*}$ is less than $\beta$ (See Appendix). We have the following proposition.

Proposition 3. The maximum equilibrium growth rate can be attained by setting the education-research allocation to $\bar{\lambda}$ and imposing zero income tax rate if $\bar{\lambda} \leq \lambda^{*}$, or by setting the education-research allocation to $\lambda^{*}$ and imposing a positive income tax rate if $\bar{\lambda}>\lambda^{*}$.

Proposition 3 gives us a role of the income tax different from Dasgupta (1999). In his model, the rise in the income tax rate imposed on capital income gives rise to a decrease in the growth rate. It is explained as follows. Since household income is only capital income in his model, the rise in the income tax rate reduces his income and saving, in turn, the rate of capital accumulation is reduced. Then, the maximum growth rate can be attained by imposing zero income tax rate. But, in our model, we cannot choose the income tax rate independently of the education-research allocation. In our setting, the income tax rate is determined by the required budget in the educational sector, which depends on the production efficiency in this sector. Since there should be a certain efficient education-research allocation, for small $\lambda$, we can increase both outputs without raising income tax revenue. However for large $\lambda$, we cannot do this without raising the income tax rate. This efficiency condition leads to our conclusion that optimal income tax rate is not zero.

Let us consider the effect of change of the educationresearch allocation on the social welfare. From (20) and (23), we have 7

$$
c(\theta)=(\rho+(\sigma-1) g) h(0) e^{g \theta} .
$$

Substituting (33) to the social welfare (4),

7 The convergence of social welfare requires $\rho+(\sigma-1) g>0$, which is assumed to hold.

$$
U=\frac{h(0)^{1-\sigma}}{1-\sigma} \frac{1}{(\rho+(\sigma-1) g)^{\sigma}} .
$$

Therefore, at the steady growth equilibrium, the social welfare rises as the equilibrium growth rate rises. From proposition 3, we obtain the following proposition.

Proposition 4. In the steady state growth equilibrium, the social welfare is maximized by setting the education-research allocation to $\bar{\lambda}$ and imposing zero income tax rate when $\bar{\lambda} \leq \lambda^{*}$, or setting the education-research allocation to $\lambda^{*}$ and imposing a positive income tax rate when $\bar{\lambda} \leq \lambda^{*}$, so as to attain maximum equilibrium growth rate.

While Dasgupta(2001) shows that the optimal income tax rate is zero, the tax is imposed on capital income of household. In the optimal tax literature, it has been discussed that the optimal long-run tax on capital income is zero on average. Judd (1999) shows that this zero-average-tax result holds also for human capital if it has no final consumption value. Our result that the optimal tax rate on human capital is not necessarily zero is caused by the education-research allocation constraint, which is different from the one of traditional optimal tax literature.

\section{Concluding Comments}

Dasgupta (1999) analyzed the steady state equilibrium in the economy where the non-rival infrastructure is produced not by profit maximizing firm but by public firm. He asserted that the agent's (symbolized by an infinitely lived representative household) welfare rises, if the growth rate rises in the degree that it covers the distortion of resources allocation. In this case, since the lower the income tax rate derives the higher the growth rate, the optimal income tax rate should be zero.

In Dasgupta (1999), the tax rate is a control variable for government. However, in our model, it is not a control variable, rather it is determined by the market equilibrium condition, depending on the education-research allocation in the educational sector. This follows from an additional constraint on the production functions of human capital and social knowledge. The technology will be given according to how the educational sector allocates human capital between education and research activities.

In our setting, while a reduction of the income tax rate raises household income and demand for human capital, it reduces the revenue of the educational sector and leads to decreased outputs and, in turn, reduces the growth rate beyond some tax rate. Therefore, the optimal income tax rate should not necessarily be zero in our economy.

We supposed in this paper that the revenues of the educational sector are financed by the income tax and the rent on the use of social knowledge rather than by the sale of education. This means that education supplied by the educational sector is free of change, which leads to a distortion in our economy. To analyze whether the market system can attain full optimum, we must consider the pricing rule of education as in Dasgupta (2001) and Furumatsu and Shirai (1998). 


\section{Appendix}

We prove here that $\lambda^{*}$ is less than $\beta$. To simplify the notation, we denote $g^{s}$ and $g^{d}$ functions as $S(\tau, \lambda)$ and $D(\tau, \lambda)$, respectively. Then,

$$
\begin{gathered}
\mathrm{S}_{\tau}=\alpha \beta \mathrm{B} \lambda^{\beta}\left[\alpha\left(\frac{1-\alpha}{\alpha}+\tau\right)\left(\frac{\lambda}{1-\lambda}\right)^{\beta}\right]^{\beta-1}>0 \\
D_{\tau}=-\frac{1}{\sigma} \alpha^{2} A((1-\tau) \alpha)^{\alpha-1}\left(\frac{\lambda}{1-\lambda}\right)^{\beta(\alpha-1)}<0 \\
\mathrm{~S}_{\lambda}=\alpha^{\beta} \beta \mathrm{B} \lambda^{\beta-1}\left(\frac{\lambda}{1-\lambda}\right)^{\beta(\beta-1)}\left(\frac{1-\alpha}{\alpha}+\tau\right)^{\beta} \frac{\beta-\lambda}{1-\lambda^{\prime}} \\
D_{\lambda}=-\frac{1}{\sigma}(1-\alpha) \beta A[(1-t) \alpha]^{\alpha}\left(\frac{\lambda}{1-\lambda}\right)^{\beta(\alpha-1)-1} \frac{1}{(1-\lambda)^{2}}<0
\end{gathered}
$$

where $S_{\iota}=\partial S / \partial \iota, D_{\iota}=\partial D / \partial \iota(\iota=\tau, \lambda)$. As easily shown, $S_{\lambda} \geq(<) 0$ as $\lambda \leq(>) \beta$. Then $g^{s}$ curve shifts upward (downward) when $\lambda<(>) \beta$.

At the equilibrium, $S(\tau, \lambda)=D(\tau, \lambda)$ holds for any $\lambda$, then we obtain by totally differentiating

$$
\frac{d \tau}{d \lambda}=\frac{D_{\lambda}-S_{\lambda}}{S_{\tau}-D_{\tau}}
$$

Since the denominator of (A.5) is positive and the numerator is negative when $\lambda \leq \beta$, the sign of (A.5) is negative when $\lambda \leq \beta .8$

Further, by using (A.5), we have

$$
\frac{d g}{d \lambda}=S_{\tau} \frac{d \tau}{d \lambda}+S_{\lambda}=\frac{S_{\tau} D_{\lambda}-S_{\lambda} D_{\tau}}{S_{\tau}-D_{\tau t}} .
$$

$\mathrm{S}_{\tau} \rightarrow 0$ and $D_{\tau} \rightarrow-\infty$ as $\lambda \rightarrow 0$. Therefore $d g / d \lambda$ is positive small $\lambda$. On the contrary, when $\lambda \geq \beta, d g / d \lambda$ is negative. Therefore, we have some value of $\lambda$ which makes $d g / d \lambda=0$ in $0<\lambda<\beta$. We denoted above this $\lambda$ as $\lambda^{*}$. Consequently, $\lambda^{*}$ which attains the maximum growth rate is bounded by the technical parameter of the production function in the educational sector.

\section{References}

[1] Dasgupta, D (1999), "Growth versus Welfare in a Model of Nonrival Infrastructure", Journal of Development Economics, Vol.58, No.2, pp.359-385.

[2] Dasgupta, D.(2001), "Lindahl Pricing, Nonrival Infrastructure and Endogenous Growth", Journal of Public Economic Theory, Vol.3, No.4, pp.413-430.

[3] Eckstein, Z and I. Zilcha (1994), "The Effects of Compulsory Schooling on Growth, Income Distribution and Welfare", Journal of Public Economics, Vol.54, pp.339-359.

[4] Furumatsu N. and M. Shirai (1998), "The Optimal Subsidies to Higher Education, with Special Reference to Education and Research", Discussion Paper (Institute of Economics, Chukyo University), No.40, pp.1-12.

[5] Judd, K.L.(1999), "Optimal Taxation and Spending in General Competitive Growth Models", Journal of Public Economics, Vol.71, pp.1-26.

[6] Kitaura, K and M. Shirai (2012), "Researches in Higher Education Institutes and Economic Growth", in Shirai M, K. Kamata and M. Yuda eds., Public Economics Studies V, Keisou Shobou (in Japanese) .

[7] Lucas, R.E. (1988), "On the Mechanics of Economic Development", Journal of Monetary Economics, Vol.22, pp.342 .

[8] Ministry of Education, Culture, Sports, Science and Technology-JAPAN(2013), Recent Trends in Education Overseas, Akashi Shoten (in Japanese) .

[9] Romer, P.M. (1990), "Endogenous Technological Change", Journal of Political Economy, Vol.98, No.5, pp.S71-S102.

[10] Zhang, J.(1996), "Optimal Public Investments in Education and Endogenous Growth", Scandinavian Journal of Economics, Vol.98, pp.387-404. 